\title{
Regularization of Newton constant, trans-Planckian dispersion relation and symmetry of particle spectrum
}

\author{
Pei-Ming Ho \\ Department of Physics, Harvard University, Cambridge, MA 02138, USA \\ and \\ Department of Physics, National Taiwan University, Taipei, Taiwan, Republic of China \\ E-mail: pmho@fas.harvard.edu
}

Received 4 December 2003

Published 26 April 2004

Online at stacks.iop.org/CQG/21/2641

DOI: $10.1088 / 0264-9381 / 21 / 11 / 009$

\begin{abstract}
We consider the possibility that the UV completeness of a fundamental theory is achieved by a modification of propagators at large momenta. We assume that general covariance is preserved at all energies, and focus on the coupling of a scalar field to the background geometry as an example. Naively, one expects that the gravitational interaction, like Yukawa interactions, will be regularized by a propagator which decays to zero sufficiently fast above some cut-off scale, but we show that in order to avoid the ultraviolet divergence, the propagator should approach a nonzero constant. This incompatibility between the regularizations of gravitational and Yukawa interactions suggests that a symmetry of the particle spectrum is needed for a UV-complete fundamental theory.
\end{abstract}

PACS numbers: 11.25.-w, 11.25.Mj, 11.25.Sq

\section{Introduction}

It is generally believed that a fundamental theory including quantum gravity should be free of divergences. In this paper, we want to study the implications of the UV finiteness of the fundamental theory. What are the properties the theory should possess in order to be UV finite? Finding answers to such questions may help us to construct the fundamental theory; and even after the fundamental theory is found, this kind of question may help us understand it better.

We will assume that the UV completeness of the fundamental theory holds perturbatively, i.e., the theory is still UV finite after turning off all interactions among the matter fields. This is obviously a technical necessity. If the UV completeness is a nonperturbative feature of the fundamental theory, we will not be able to derive any implication from it until we know the exact nonperturbative definition of the theory. 
As a first step towards understanding what is needed for the quantum gravity to be UV finite, we consider the gravitational coupling of a free scalar field to the background geometry. With the standard minimal coupling to a curved spacetime, the quantum loop diagrams diverge, and we would like to know what kind of modification to the standard theory will regularize it.

More specifically, in a curved background, the path integral of a matter field $\phi$ generates a Hilbert-Einstein term in its effective action. This leads to a quantum correction to the Newton constant

$$
G^{-1}=G_{0}^{-1}+\delta G^{-1},
$$

where $G_{0}$ is the bare coupling constant, and $\delta G^{-1}$ is the coefficient of the Hilbert-Einstein term in the effective action. In the theory of induced gravity [1], $G_{0}^{-1}=0$, and the full Newton constant arises as quantum corrections. With the standard coupling of matter fields to gravity, $\delta G^{-1}$ diverges and needs to be regularized.

In a UV-complete theory, both the bare coupling $G_{0}^{-1}$ and the quantum correction should be finite. In the following, we will derive constraints on the unknown fundamental theory by requiring $\delta G^{-1}$ to be finite.

A matter field's quantum contribution to the Newton constant is closely related to the blackhole entropy [2]. Previous works [3-5] propose to suitably modify dispersion relations at high energies to regularize the black-hole entropy ${ }^{1}$. Hypothetical relations between Hamiltonian and momentum can make the Newton constant finite, but its obvious disadvantage is that general covariance is broken. We would like to preserve the general covariance as an exact symmetry although it is logically possible that it is only an approximate symmetry at low energies. Another technical disadvantage of modifying dispersion relations is that, as a result of the loss of general covariance, one needs to propose dispersion relations separately for each class of backgrounds, and calculations may need to be carried out independently for each case.

Instead of modifying the dispersion relation, we consider modification to the Lagrangian by higher derivatives. At the zeroth-order approximation with respect to all other interactions, the Lagrangian of a scalar field is quadratic, and is characterized by the corresponding propagator. The finiteness of the Newton constant imposes constraints on the propagator, assuming that the quantum contribution of each field is finite. Remarkably, our calculation shows that the propagator has to approach a nonvanishing constant in the limit of large momentum $k^{2} \rightarrow \infty$.

This is in contrast to the situation for Yukawa-type interactions in which the propagator needs to approach zero fast enough as $k^{2} \rightarrow \infty$, so that Feynman loop diagrams converge. Because of this difference between gravitational and Yukawa interactions, one cannot make both Newton constant and Yukawa couplings finite by propagator modification.

Hence we are forced to re-examine our assumptions. A possibility is that some symmetry among all matter fields, such as supersymmetry, ensures that the infinities coming from different fields cancel each other. A more complete set of alternatives will be examined in section 3 .

\section{One-loop calculation}

In the zeroth-order approximation with respect to all couplings except the gravitational coupling to the background geometry, the action of the fundamental theory is a sum of

\footnotetext{
1 An exception is [6] in which the Pauli-Villars regularization was used to calculate the Hawking radiation.
} 
free field actions for each particle field. For a scalar field, the action is of the form

$$
S=\frac{1}{2} \int \mathrm{d}^{d} x \phi \mathcal{D} \phi
$$

where $\mathcal{D}$ is a pseudodifferential operator. The effective action $W$ is defined by the path integral

$$
\mathrm{e}^{-W}=\int D \phi \mathrm{e}^{\frac{1}{2} \int \mathrm{d}^{d} x \phi \mathcal{D} \phi} .
$$

Note that $W$ is defined up to an (infinite) constant in the above. Apart from this constant, terms involving physical interactions should be finite in a UV-complete theory.

On a slightly curved space, one can express the effective action as a power expansion of the spacetime curvature

$$
W=\delta \Lambda_{0} \int \mathrm{d} V+\delta \lambda_{0} \int \mathrm{d} \sigma+\delta G^{-1}\left(\int \mathrm{d} V R-2 \int \mathrm{d} \sigma K\right)+\cdots,
$$

where $\mathrm{d} \sigma$ is the volume element on the boundary of the four-dimensional spacetime, and $K$ is the curvature on the boundary.

Due to general covariance, the effective action $W$ is always of the form (4) in the weak curvature expansion, with possibly different coefficients. Terms involving higher orders of the curvature tensor are omitted in (4). Coefficients of higher-order terms are weighted by higher inverse powers of a certain effective UV cut-off energy scale $M$. Equation (4) is a low-energy expansion which makes sense only if the curvature is much smaller than $M^{2}$.

The first two terms in (4) correspond to the quantum contribution of $\phi$ to the cosmological constant in the bulk and on the boundary. The rest are the lowest order generally invariant terms in the weak curvature expansion, and they contribute to the Newton constant. The standard choice of $\mathcal{D}$ in (2) is the Laplace operator for a massless scalar field, for which all the coefficients in $W$ are UV divergent.

The boundary term $\int K$ in (4) has to be present for the following reason. In the canonical formulation, the Lagrangian should be a function of the dynamical variables and their first derivatives. Since the curvature $R$ involves second derivatives of the metric, we should apply integration by parts to the bulk term so that it only has first derivatives. The boundary term in (4) then cancels the boundary term generated by integration by parts, so that the full action is of the standard form. For this reason the coefficient of the boundary term is determined by that of the bulk term. The fact that the ratio of the coefficients is fixed has very significant physical implications. It provides a low-energy argument [7] for the compatibility between the renormalization of the Newton constant and that of the Bekenstein-Hawking entropy [2].

Let us digress a little to explain this point as it will also shed light on possible applications of the results of this paper to the black-hole entropy. As we just saw, quantum fluctuations of a matter field contribute to the Newton constant. At the same time it contributes to the black-hole entropy. The Bekenstein-Hawking relation

$$
S=\frac{A}{4 G}
$$

is therefore corrected on both sides. In [2] it was suggested that the renormalized entropy and the renormalized Newton constant always cooperate to keep (5) intact in the low-energy limit. A low-energy argument for this claim was given in [7] as follows. We saw above that the low-energy effective action (4) determines the quantum correction to the Newton constant. In fact $W$ also determines the entropy, at least according to one of its definitions. For a black-hole background, as a vacuum solution to Einstein gravity, the Hilbert-Einstein term in (4) vanishes, and $W$ is thus determined by the boundary term. Hence the entropy is proportional to the coefficient of the boundary term in $W$, while the Newton constant to the 
bulk term coefficient. The fixed ratio of the coefficients is thus translated to the fixed ratio of the renormalization of $S$ and $G^{-1}$. This connection between the quantum correction of the Newton constant and that of the black-hole entropy is part of the motivation of this work.

Now we calculate the quantum correction to the Newton constant due to a scalar field with the action (2). In the fundamental theory, the operator $\mathcal{D}$ should produce finite coefficients in $W$. The most general quadratic generally covariant Lagrangian for $\phi$ allowing higher derivatives is

$$
\mathcal{L}=\phi f\left(-\nabla^{2}\right) \phi .
$$

Although higher derivatives are notorious for introducing various problems to the canonical formulation, from the viewpoint of effective theories, the proper way to deal with higher derivatives is to treat it perturbatively [8-10], and various problems may be resolved.

The gravitational coupling of the scalar field to the background geometry is hidden in the inverse propagator

$$
f\left(-\nabla^{2}\right)=f\left(-\partial^{2}\right)+\mathcal{F}+\cdots,
$$

where $\mathcal{F}$ is the first-order term in the weak curvature expansion. At this point we can already see the origin of the drastic difference between gravitational coupling and Yukawa coupling. While Yukawa coupling is independent of the propagator, the gravitational coupling is determined by the propagator because of the requirement of general covariance. A propagator that decays to zero sufficiently fast over some energy scale effectively cuts off the integration over momentum for a Feynman diagram. This helps the Yukawa coupling to be UV finite. However, if the propagator is modified to have steeper slopes, the gravitational coupling $\mathcal{F}$ is enhanced, and it is not clear whether the Feynman integral will be more divergent or less divergent. We need to calculate the loop diagrams explicitly to decide.

An advantage of our approach is that since the calculation is generally covariant, we do not have to calculate the Newton constant for every background. We can choose any background with nontrivial curvature. This is because the lowest-order term in the weak curvature expansion (4) is uniquely given by the Hilbert-Einstein action. In contrast, terms of higher orders in the curvature tensor are not unique. To determine their coefficients we will need to do the same calculation for several different backgrounds.

For simplicity, we choose the background to be a (large) sphere $S^{d}$. In normal coordinates, the metric of a sphere is

$$
g_{i j}=\left(1+\alpha x^{2}\right) \delta_{i j}+\cdots
$$

in the neighbourhood of the north pole. The parameter $\alpha$ is proportional to the constant curvature on the sphere. One can easily check that

$$
\nabla^{2}=\frac{1}{\sqrt{g}} \partial_{i} \sqrt{g} g^{i j} \partial_{j}=\partial^{2}+\Delta
$$

where

$$
\Delta=\alpha\left((d-2) x \cdot \partial-x^{2} \partial^{2}\right)+\cdots .
$$

Using the identity

$$
\left[\left(\partial^{2}\right)^{n}, \Delta\right]=-4 n \alpha(n+x \cdot \partial)\left(\partial^{2}\right)^{n},
$$

one can show that for a generic $f\left(-\nabla^{2}\right), \mathcal{F}$ defined by $(7)$ is of the form

$$
\mathcal{F}=\mathcal{F}_{0}\left(-\partial^{2}\right)+(x \cdot \partial) \mathcal{F}_{1}\left(-\partial^{2}\right)+x^{2} \mathcal{F}_{2}\left(-\partial^{2}\right) .
$$

We will only need the expressions of $\mathcal{F}_{0}$ and $\mathcal{F}_{2}$ below. For any smooth function $f$,

$$
\mathcal{F}_{0}(y)=\alpha\left(2 y^{2} \frac{\mathrm{d}^{3}}{\mathrm{~d} y^{3}}+3 y \frac{\mathrm{d}^{2}}{\mathrm{~d} y^{2}}\right) f(y)=2 \alpha y^{-1 / 2} \frac{\mathrm{d}}{\mathrm{d} y}\left(y^{3 / 2} \frac{\mathrm{d}^{2}}{\mathrm{~d} y^{2}} f(y)\right),
$$




$$
\mathcal{F}_{2}(y)=\alpha y \frac{\mathrm{d}}{\mathrm{d} y} f(y)
$$

where we have denoted $k^{2}$ by $y$. The $\mathcal{F}_{1}$ term does not contribute because it is odd under $k \rightarrow-k$ and we will integrate over $k$ for the one-loop diagram.

It is straightforward to find the one-loop effective action

$$
W=\frac{1}{2} \int \mathrm{d}^{d} x \int \frac{\mathrm{d}^{d} k}{(2 \pi)^{d}} \frac{\mathcal{F}_{0}\left(k^{2}\right)+x^{2} \mathcal{F}_{2}\left(k^{2}\right)}{f\left(k^{2}\right)}+\text { constant. }
$$

The first term is the Hilbert Einstein term as it is proportional to $\alpha$, which is proportional to the constant curvature. The contribution of quantum fluctuations of $\phi$ to the Newton constant is thus proportional to

$$
\int \mathrm{d}^{d} x\left(w+x^{2} v\right)
$$

where

$$
w=\int \mathrm{d} y y^{\frac{d-2}{2}} \frac{\mathcal{F}_{0}(y)}{f(y)}, \quad v=\int \mathrm{d} y y^{\frac{d-2}{2}} \frac{\mathcal{F}_{2}(y)}{f(y)} .
$$

For this quantum correction to be finite, we need both $w$ and $v$ to be finite.

We check the finiteness of $w$ and $v$ by dividing the integral over $y=k^{2}$

$$
w=w_{0}+w_{1}+w_{\infty}, \quad v=v_{0}+v_{1}+v_{\infty},
$$

to the region of small $y$, the region of finite $y$ and the region of infinite $y$. The middle region usually contributes a finite number to the Newton constant for a well-defined $f(y)$. We need to examine the small $y$ region and large $y$ region more carefully.

For the integral over $y$ around $y=0$, we can approximate $f(y)$ by the canonical expression

$$
f(y) \simeq y,
$$

and find

$$
w_{0}=0, \quad v_{0} \propto \int_{0} \mathrm{~d} y y^{\frac{d-2}{2}},
$$

which is finite if $d \geqslant 2$.

For the integral over $y$ to $y \rightarrow \infty$, we need to examine various asymptotic behaviours of $f(y)$. Assuming that $f(y) \propto y^{n}$ for large $y$, we find

$$
w_{\infty} \propto \int^{\infty} \mathrm{d} y y^{\frac{d-4}{2}}, \quad v_{\infty} \propto \int^{\infty} \mathrm{d} y y^{\frac{d-2}{2}} .
$$

The expressions above are not valid if $n=0,1 / 2$ or 1 , in which case $w_{\infty}$ vanishes according to (13). Similarly $v$ vanishes if $n=0$ according to (14). Therefore, the only case with finite quantum correction to the Newton constant is $n=0$. That is, we need

$$
f\left(k^{2}\right) \rightarrow \text { constant } \quad \text { as } \quad k^{2} \rightarrow \infty .
$$

In addition, this constant has to be nonzero for $w$ and $v$ to be finite.

One can try a more general ansatz for $f(y)$ such as

$$
f(y)=y^{n} \mathrm{e}^{\beta y},
$$

but we will only arrive at the same conclusion that we need $f$ to approach a nonzero constant. Although 0 is also a constant and $\mathcal{F} \rightarrow 0$ if $f \rightarrow 0$, the factor $y^{(d-2) / 2}$ in $v_{\infty}(21)$ keeps us from finding examples for which $v_{\infty}$ is finite while $f \rightarrow 0$. 
Examples of inverse propagators which give finite quantum contribution to the Newton constant include

$$
\begin{aligned}
& f\left(-\nabla^{2}\right)=\frac{M^{2}\left(-\nabla^{2}+m^{2}\right)}{-\nabla^{2}+M^{2}}, \\
& f\left(-\nabla^{2}\right)=M^{2}\left(1-\mathrm{e}^{\nabla^{2} / M^{2}}\right)+m^{2}, \\
& f\left(-\nabla^{2}\right)=-\nabla^{2} \mathrm{e}^{\nabla^{2} / M^{2}}+m^{2},
\end{aligned}
$$

where $m$ is a constant determining the low-energy effective mass of $\phi$, and $M$ is the fundamental energy at which the wave equation is significantly modified.

These examples are characterized by two energy scales. First, there is a scale $M$ beyond which the propagator is roughly a constant. It is the effective UV cut-off for gravitational interaction. The other scale, which is $M, \sqrt{M^{2}+m^{2}}$ and $m$ for the three examples, respectively, specifies the constant value of the propagator in the high-energy limit. This scale characterizes the scalar's coupling to an ordinary source term in the high-energy limit ${ }^{2}$.

The desirable properties satisfied by the examples above are

1. the wave equation is covariant;

2. the low-energy wave equation is canonical, i.e., $f\left(k^{2}\right) \rightarrow\left(k^{2}+m_{0}^{2}\right)$ for small $k$;

3. the contribution of quantum fluctuation to the Newton constant is finite.

What we see here is quite surprising at first sight. Naively, people expect that the quantum loop calculation is UV finite if we impose a cut-off on the propagator, that is, if the propagator goes to zero quickly above some scale $k^{2}=\Lambda^{2}$. Our calculation shows however that the UV finiteness of the Newton constant requires instead that the propagator $f^{-1}\left(k^{2}\right)$ approaches to a nonvanishing constant.

As we commented above, this is due to the fact that gravitational coupling is determined by the propagator. When we turn on a curvature in the background, the new interaction term $\phi \mathcal{F} \phi$ appearing in the Lagrangian is larger when $f\left(k^{2}\right)$ varies faster with $k^{2}$, because $\mathcal{F}$ is roughly proportional to the derivative of $f$. The exponential decay factor in (23), for example, will make both the denominator $f$ and the numerator $\mathcal{F}$ to increase exponentially with $y$ in the expressions of $w$ and $v$. It is therefore not helpful to have the propagator $f^{-1}$ to vanish quickly above some scale. At high energies, these propagators decouple from gravity, but couple to ordinary source terms much stronger than ordinary propagators.

Without any calculation, one can easily see that if the propagator is a constant, the field is decoupled from gravity. Our calculation shows that this extreme decoupling is needed at high energies for the UV convergence of gravitational interaction. Once this conclusion is made, we expect that all higher-order terms in the curvature expansion of the effective action will also be finite.

As an aside, since gauge field interactions are also encoded in the kinetic term of a scalar field, a propagator that approaches to a nonzero constant also defines a UV finite gauge field interaction.

2 An ordinary source is introduced in the Lagrangian (6) by adding another term $-\phi j$. The equation of motion is then modified to

$$
f\left(-\nabla^{2}\right) \phi=j .
$$

In the high-energy limit the solution of $\phi$ for a given source $j$ is simply given by

$$
\phi \simeq \text { constant } j .
$$




\section{Remarks}

The main result of the previous section is that the propagator has to approach a nonzero constant for the Newton constant to be UV finite. By itself the result can have many applications to highenergy physics and cosmology. Here we discuss its implications on the logical possibilities for a UV-complete theory of quantum gravity.

\section{1. $f \rightarrow$ constant:}

In this case, the Newton constant is finite for any field content in the free field limit of the theory.

(a) If there is Yukawa coupling in the theory, one needs a mechanism, possibly a large symmetry among all the fields, to make loop diagrams involving Yukawa interactions finite.

(b) There is no Yukawa coupling in the theory. The Yukawa coupling in the standard model arises from other types of interactions at high energies.

\section{2. $f \rightarrow \infty$ :}

In this case we need a symmetry, such as supersymmetry, among the field content of the fundamental theory to cancel each field's contribution to the Newton constant. It will be interesting to calculate higher-order terms in the effective action $W$. A tempting scenario is where infinitely many constraints are arrived at and the spectrum is uniquely fixed.

3. Neither of the above:

A symmetry or symmetries among all the fields of the theory is responsible for making both gravitational interaction and other interactions UV finite.

Except possibility (1a), all possibilities above imply the necessity of a symmetry among the particles of the theory. An inventive reader might also point out other possibilities. Another simple possibility which admits a finite Newton constant without requiring the propagator to approach a constant is to add nonminimal couplings to $R$ for some scalar fields. However, a perfect cancellation of the divergences from all species by the nonminimal coupling constants for part of the particle spectrum is unnatural without a symmetry.

Furthermore, unless the propagator goes to a nonzero constant, the UV problem for gravitational coupling becomes more serious when we consider higher-order terms in the curvature expansion of the effective action. As we go to higher and higher-order terms, we will need to fine-tune more and more couplings. This is apparently highly unnatural.

In contrast, if the propagator approaches a constant, we can easily argue that all higherorder terms will also be finite because in the high-energy limit the free field Lagrangian is simply decoupled from the background geometry.

Possibility (1) seems to provide the simplest way to construct a UV finite theory of quantum gravity. A toy model can be built on two scalar fields. Gravity is induced by integrating out one of the scalar fields. Then we obtain a UV finite quantum gravity coupled to a single scalar field. Such toy models may be useful in the future to understand conceptual puzzles about quantum gravity.

Another remarkable property of possibility (1) is that the large $k$ behaviour of the propagator is almost completely determined. This kind of information for trans-Planckian physics is very precious and can be applied to cosmological models for the very early universe. Possible effects of higher derivatives in cosmology have been discussed in [11-14].

For possibility (2), the finiteness of the Newton constant leads to a strong constraint on this symmetry that we need. For example, the contribution of minimally coupled fields of 
spin $0,1 / 2$ and 1 to the Newton constant has been calculated [15] as

$$
\delta G^{-1}= \begin{cases}\frac{1}{12 \pi} \frac{1}{\epsilon^{2}} & \text { spin } 0, \\ \frac{s}{24 \pi} \frac{1}{\epsilon^{2}} & \text { spin } 1 / 2, \\ \frac{d-8}{12 \pi} \frac{1}{\epsilon^{2}} & \text { spin } 1,\end{cases}
$$

where $s$ is the dimension of fermion representation ${ }^{3}$, and $\epsilon$ is a parameter of regularization. For a field theory in which no fields of higher spin are present (gravity is induced), we need

$$
N_{0}+\frac{s}{2} N_{1 / 2}+(d-8) N_{1}=0,
$$

where $N_{0}, N_{1 / 2}$ and $N_{1}$ are the number of fields of spin $0,1 / 2$ and 1 , respectively. Obviously this is impossible if $d \geqslant 8$, in which case fields of higher spins are needed.

String theory is classified as possibility (2). In Witten's bosonic open string field theory [16], all fields have the canonical kinetic term, and they appear in the cubic interaction terms in the form of

$$
\tilde{\phi}=\mathrm{e}^{a^{2} \partial^{2}} \phi,
$$

where $a^{2}=\ln (3 \sqrt{3} / 4) \alpha^{\prime}$. Similarly, in the bosonic closed string field theory we have $a^{2}=$ $\frac{1}{2} \ln (3 \sqrt{3} / 4) \alpha^{\prime}$ [17]. We can rewrite the action in terms of $\tilde{\phi}$, and then the interactions are Yukawa couplings. The kinetic term now gets an exponential factor of derivatives. The quadratic part of the Lagrangian is $\phi \mathcal{D} \phi$ with $\mathcal{D}$ of the form

$$
\mathcal{D}=\left(k^{2}+m^{2}\right) \mathrm{e}^{2 a^{2} k^{2}} .
$$

This gives a propagator which exponentially decays to zero at large $k^{2}$, and is good for making Feynman loop diagrams finite. But its quantum contribution to the Newton constant diverges. The conclusion is again that there is a conspiracy among the different species in the particle spectrum of string theory, a picture consistent with the widespread belief that there is a big symmetry yet to be understood [18].

String theory also serves as an example in which our assumption of a zeroth-order approximation is valid. Although string theory has a universal coupling constant that determines all couplings, one can turn on the background and turn off the string coupling at the same time such that in a double scaling limit the coupling to the background geometry is finite. A well-known example is the anti de Sitter space with RR flux. The radius of the anti de Sitter space is determined by the flux $N$ and the string coupling constant $g_{s}$. One can take the limit $g_{s} \rightarrow 0$ and $N \rightarrow \infty$ simultaneously such that the radius is fixed and large.

\section{Acknowledgments}

The author thanks Chong-Sun Chu, Herbert Fried, Jong-Ping Hsu, Kyungsik Kang, Hsien-chung Kao, Albion Lawrence, Feng-Li Lin, Horatiu Nastase, Sanjaye Ramgoolam, Andrew Strominger and Nicolaos Toumbas for helpful discussions. This work is supported in part by the National Science Council, the National Center for Theoretical Sciences, the CosPA project of the Ministry of Education, Taiwan, Republic of China and the Center for Theoretical Physics at the National Taiwan University.

${ }^{3}$ For instance, $s=2$ for a Weyl spinor in four dimensions. 


\section{References}

[1] Sakharov A D 1968 And the theory of gravitation Sov. Phys. Dokl. 121040

Sakharov A D 1967 Dokl. Akad. Nauk Ser. Fiz. 17770 (SOPUA,34,394.1991; GRGVA,32,365-367.2000)

[2] Susskind L and Uglum J 1994 Phys. Rev. D 502700 (Preprint hep-th/9401070)

[3] Unruh W G 1995 Phys. Rev. D 512827

[4] Corley S and Jacobson T 1996 Phys. Rev. D 541568 (Preprint hep-th/9601073)

[5] Chang D, Chu C S and Lin F L 2003 Preprint hep-th/0306055

[6] Hambli N and Burgess C P 1996 Phys. Rev. D 535717 (Preprint hep-th/9510159)

[7] Larsen F and Wilczek F 1996 Nucl. Phys. B 458249 (Preprint hep-th/9506066)

[8] Yang C N and Feldman D 1950 Phys. Rev. 79972

[9] Cheng T C, Ho P M and Yeh M C 2002 Nucl. Phys. B 625151 (Preprint hep-th/0111160)

[10] Cheng T C, Ho P M and Yeh M C 2002 Phys. Rev. D 66085015 (Preprint hep-th/0206077)

[11] Gu J A, Ho P M and Ramgoolam S 2001 Preprint hep-th/0101058

[12] Brandenberger R and Ho P M 2002 Phys. Rev. D 66023517

Brandenberger R and Ho P M 2002 AAPPS Bull. 1210 (Preprint hep-th/0203119)

[13] Huang Q G and Li M 2003 J. High Energy Phys. JHEP 06(2003)014 (Preprint hep-th/0304203) Huang Q G and Li M 2003 Preprint astro-ph/0308458

[14] Tsujikawa S, Maartens R and Brandenberger R 2003 Preprint astro-ph/0308169

[15] Kabat D 1995 Nucl. Phys. B 453281 (Preprint hep-th/9503016)

[16] Witten E 1986 Nucl. Phys. B 268253

[17] Kostelecky V A and Samuel S 1990 Phys. Rev. D 421289

[18] Gross D J and Mende P F 1987 Phys. Lett. B 197129 